Homology, Homotopy and Applications, vol.4(2), 2002, pp.277-294

\title{
RESOLUTIONS BY MAPPING CONES
}

\author{
JÜRGEN HERZOG AND YUKIHIDE TAKAYAMA
}

\author{
(communicated by Clas Löfwall)
}

\begin{abstract}
Resolutions that arise as iterated mapping cones are considered. Explicit resolutions are given for monomial ideals with linear quotients which admit decomposition functions, and it is studied under which conditions a mapping cone admits a DG algebra structure.
\end{abstract}

\section{To Jan-Erik Roos on his sixty-fifth birthday}

\section{Introduction}

Many well-known free resolutions arise as iterated mapping cones. Prominent examples are the Eliahou-Kervaire resolution of stable monomial ideals (as noted by Evans and Charalambous [10]), and the Taylor resolution. The idea of the iterated mapping cone construction is the following: Let $I \subset R$ be an ideal generated by $f_{1}, \ldots, f_{n}$, and set $I_{j}=\left(f_{1}, \ldots, f_{j}\right)$. Then for $j=1, \ldots, n$ there are exact sequences

$$
0 \longrightarrow R /\left(I_{j-1}: f_{j}\right) \longrightarrow R / I_{j-1} \longrightarrow R / I_{j} \longrightarrow 0 .
$$

Assuming that the free $R$-resolution $F$ of $R / I_{j-1}$ is already known, and a free $R$-resolution $G$ of $R /\left(I_{j-1}: f_{j}\right)$ is also known, one obtains a resolution of $R / I_{j}$ as a mapping cone of a complex homomorphism $\psi: G \rightarrow F$ which is a lifting of $R /\left(I_{j-1}: f_{j}\right) \rightarrow R / I_{j-1}$. Of course one cannot expect that such a resolution will be minimal in general. However this construction yields an inductive procedure to compute a resolution of $R / I$ provided for each $j$, a resolution of $R /\left(I_{j-1}: f_{j}\right)$ is known as well as the comparison map $\psi$.

So it is natural to consider classes of ideals for which the colon ideals $I_{j-1}: f_{j}$ are generated by regular sequences. But even in this nice case it is still hard to construct the comparison maps. In the first section of this paper we therefore restrict ourselves to the case that $I$ is a monomial ideal in a polynomial ring, and that the colon ideals in question are generated by subsets of the variables. In this case we say that $I$ has linear quotients. At a first glance these hypotheses seem to be very restrictive. On the other hand, there are many interesting examples of such ideals. All stable and squarefree stable ideals belong to this class, as well as all matroidal ideals (see Section 1 for the definitions).

Received January 8, 2001; published on July 12, 2002.

2000 Mathematics Subject Classification: 13D02, 13C13.

Key words and phrases: Free resolutions, monomial ideals, mapping cones, DG algebras.

(c) 2002, Jürgen Herzog and Yukihide Takayama. Permission to copy for private use granted. 
It is easy to see that $I$ has linear quotients, if and only if the first syzygy module of $I$ has a quadratic Gröbner basis, which, in case of a Stanley ideal $I_{\Delta}$ attached to the simplicial complex $\Delta$, is equivalent to saying that the Alexander dual $\Delta^{*}$ of $\Delta$ is nonpure shellable. This fact was communicated to us by Sköldberg [15]. It is also easy to see that $I$ has linear quotients if and only if $I$ satisfies condition (4.1) of Batzies and Welker [3], a condition which the authors call shellable.

It is clear that our approach only requires to describe the comparison maps in order to compute explicit free resolutions of ideals with linear quotients as iterated mapping cones. Our description of the comparison maps is modeled after Eliahou and Kervaire and is based on decomposition functions. A function $g$ which assigns to each monomial in $I$ (in a natural way) a monomial generator of $I$, see 1.7 , is called a decomposition function. If it satisfies a certain additional condition which is described in Definition 1.9, then we call it regular. Stable and squarefree stable ideals have regular decomposition functions, but also matroidal ideals as we show in Theorem 1.10. The main result of Section 1 however is Theorem 1.12 in which we give an explicit resolution of all ideals with linear quotients which admit a regular decomposition function. These include then of course also matroidal ideals for which explicit resolutions in different terms are already known by Reiner and Welker [14], and Novik, Postnikov and Sturmfels [13].

In the second part of this paper we ask ourselves under which circumstances a mapping cone can be given the structure of a DG algebra. The natural way of doing this, is to assume that $F$ (notation as above) is already a DG algebra, that $G$ is a DG $F$-module, and that the mapping cone of $\psi$ is a trivial extension of $F$ by $G$ in the category of DG modules. This idea was first used by Levin and Avramov [12] in order to compute the Poincaré series of Gorenstein algebra modulo its socle. In Section 2 we analyze under which conditions the mapping cone can be given the structure of a trivial extension and in the final Section 3 we describe cases for which resolutions which are constructed as iterated mapping cones admit a DG algebra resolution. We call such resolutions of Koszul type and show in Example 3.6 that the known DG algebra structure (see [9] and [11]) on the Taylor complex is of Koszul type, as well as the resolution of an almost complete intersection which is directly linked to a complete intersection, see Example 3.7.

\section{Monomial Ideals with linear quotients}

Let $K$ be a field, $R=K\left[x_{1}, \ldots, x_{n}\right]$ be the polynomial ring in $n$ indeterminates, and $I \subset R$ a monomial ideal. The unique minimal set of monomial generators of $I$ will be denoted by $G(I)$. The ideal $I$ is said to have linear quotients if for some order $u_{1}, \ldots, u_{m}$ of the elements of $G(I)$ and all $j=1, \ldots, m$ the colon ideals $\left(u_{1}, \ldots, u_{j-1}\right): u_{j}$ are generated by a subset of $\left\{x_{1}, \ldots, x_{n}\right\}$.

We define

$$
\operatorname{set}\left(u_{j}\right)=\left\{k \in[n]: x_{k} \in\left(u_{1}, \ldots, u_{j-1}\right): u_{j}\right\} \quad \text { for } \quad j=1, \ldots, m .
$$

Example 1.1. According to Eliahou-Kervaire [8], a monomial ideal $I$ is called stable if for all $u \in G(I)$ and all $i \leqslant m(u)$ one has that $x_{i}\left(u / x_{m(u))}\right) \in I$. Here $m(u)=\max \{i: i \in \operatorname{supp}(u)\}$, and $\operatorname{supp}(u)=\left\{i: x_{i}\right.$ divides $\left.u\right\}$. 
Let $G(I)=\left\{u_{1}, \ldots, u_{m}\right\}$, where $u_{1}>u_{2}>\cdots>u_{m}$ in the reverse degree lexicographical order with regard to $x_{1}>x_{2}>\ldots>x_{n}$. It is easy to see that $I$ has linear quotient for this order of the generators, and that $\operatorname{set}(u)=\{1, \ldots, m(u)-1\}$ for all $u \in G(I)$.

Example 1.2. In $[2]$ a squarefree monomial ideal $I$ is called squarefree stable if for all $u \in G(I)$ and all $i<m(u)$ with $i \notin \operatorname{supp}(u)$ one has that $x_{i}\left(u / x_{m(u))}\right) \in I$. With respect to the reverse degree lexicographical order on the generators, $I$ has linear quotients and $\operatorname{set}(u)=\{i: i<m(u), i \notin \operatorname{supp}(u)\}$.

We now want to analyze more carefully when a squarefree monomial ideal $I$ has linear quotients. Let $\Delta$ the corresponding simplicial complex on the vertex set $[n]=\{1, \ldots, n\}$, so that $I=I_{\Delta}$. The Alexander dual of $\Delta$ is the simplicial complex $\Delta^{*}=\{\sigma \in[n]:[n] \backslash \sigma \notin \Delta\}$. The following two statements are almost tautologically equivalent: (i) $I_{\Delta}$ has linear quotients, (ii) $\Delta^{*}$ is shellable in the non-pure sense of Björner and Wachs [5].

In particular, it follows that if the simplicial complex $\Gamma$ generated by

$$
\mathcal{B}=\left\{\operatorname{supp}(u): u \in G\left(I_{\Delta}\right)\right\}
$$

is a matroid (in which case we say that $I_{\Delta}$ is matroidal), then $I_{\Delta}$ has linear quotients. In fact, if $\Gamma$ is a matroid, then $\mathcal{B}$ is the matroid basis, and $\Delta^{*}$ is the dual matroid of $\Gamma$ with basis $\left\{[n] \backslash \operatorname{supp}(u): u \in G\left(I_{\Delta}\right)\right\}$. On the other hand, it is known that all matroids are shellable, see [4].

For the convenience of the reader we show directly that, for a simplicial complex $\Delta$, the Stanley-Reisner ideal $I_{\Delta}$ has linear quotients, if it is matroidal. If $I_{\Delta}$ is matroidal, then for all $u, v \in G\left(I_{\Delta}\right)$ and all $i \in \operatorname{supp}(u) \backslash \operatorname{supp}(v)$ there exists $j \in \operatorname{supp}(v) \backslash \operatorname{supp}(u)$ such that $x_{j}\left(u / x_{i}\right) \in I_{\Delta}$. We now claim that the generators of $I_{\Delta}$ in reverse lexicographical order have linear quotients.

We will prove a slightly more general result from which this claim will follow. Let $u=x_{1}^{a_{1}} \cdots x_{n}^{a_{n}}$ be a monomial. We set $\nu_{i}(u)=a_{i}$ for $i=1, \ldots, n$.

Lemma 1.3. Let I be a monomial ideal for which all generators have the same degree. Suppose I satisfies the following exchange property:

For all $u, v \in G(I)$ and all $i$ with $\nu_{i}(u)>\nu_{i}(v)$, there exists an integer $j$ with $\nu_{j}(v)>\nu_{j}(u)$ such that $x_{j}\left(u / x_{i}\right) \in G(I)$.

Then I has linear quotients with respect to the reverse lexicographic order of the generators.

Note that a squarefree monomial ideal is matroidal if and only if it satisfies the exchange property of Lemma 1.3. Blum [6] has shown that ideals whose generators correspond to the basis of a polymatroid satisfy the exchange property of Lemma 1.3.

Proof of 1.3. Let $u \in G(I)$, and let $J$ be the ideal generated by all $v \in G(I)$ with $v>u$ (in the reverse lexicographical order). Then

$$
J: u=(v /[v, u]: v \in J),
$$


where $[v, u]$ denotes the greatest common divisor of $v$ and $u$. Thus in order to prove that $J: u$ is generated by monomials of degree 1 , we have to show that for each $v>u$ there exists $x_{j} \in J: u$ such that $x_{j}$ divides $v /[v, u]$.

In fact, let $u=x_{1}^{a_{1}} \cdots x_{n}^{a_{n}}$ and $v=x_{1}^{b_{1}} \cdots x_{n}^{b_{n}}$. Since $v>u$, there exists an integer $i$ such that $a_{k}=b_{k}$ for $k=i+1, \ldots, n$, and $a_{i}>b_{i}$, and hence an integer $j$ with $b_{j}>a_{j}$ such that $u^{\prime}=x_{j}\left(u / x_{i}\right) \in I$. Since $j<i$, we see that $u^{\prime} \in J$, and from the equation $x_{i} u^{\prime}=x_{j} u$ we deduce that $x_{j} \in J: u$. Finally, since $\nu_{j}(v /[u, v])=$ $b_{j}-\min \left\{b_{j}, a_{j}\right\}=b_{j}-a_{j}>0$, we have that $x_{j}$ divides $v /[v, u]$.

Since for a matroid $\Delta^{*}$, the Stanley-Reisner ideal $I_{\Delta}$ has linear quotients with respect to the reverse lexicographical order of the generators, it follows that

$$
\operatorname{set}(u) \subset\{i: i<m(u), i \notin \operatorname{supp}(u)\}
$$

for all $u \in G\left(I_{\Delta}\right)$. More precisely, we have

$$
\operatorname{set}(u)=\left\{i: \operatorname{supp}(v) \backslash \operatorname{supp}(u)=\{i\} \quad \text { for some } \quad v \in G\left(I_{\Delta}\right), v>u\right\} .
$$

Example 1.4. Let $A_{1}, \ldots, A_{r}$ be non-empty subsets of $[n]$. The collection of subsets $\left\{i_{1}, \ldots, i_{r}\right\}$ of $[n]$ with $i_{j} \in A_{j}$ for $j=1, \ldots, r$ and $i_{j} \neq i_{k}$ for $j \neq k$, is the basis of a matriod, called transversal. Let $I$ be the squarefree monomial ideal whose generators correspond to the basis of this transversal matriod. Let $u \in G(I), u=x_{i_{1}} \cdots x_{i_{r}}$. The above description of $\operatorname{set}(u)$ yields in this case

$$
\operatorname{set}(u)=\bigcup_{j=1}^{r}\left\{k \in A_{j}: k \leqslant j\right\} \backslash \operatorname{supp}(u) .
$$

Let $\psi: A \rightarrow B$ be a complex homomorphism. Recall that the mapping cone of $\psi$ is the complex $C(\psi)$ with $C(\psi)_{i}=B_{i} \oplus A_{i-1}$ for all $i$, and chain map $d$ with $d_{i}: C(\psi)_{i} \rightarrow C(\psi)_{i-1}, d_{i}(b, a)=(\psi(a)+\partial(b),-\partial(a))$.

We want to apply this concept in the following situation: Suppose that $I$ has linear quotients with respect the order $u_{1}, \ldots, u_{m}$ of the generators of $I$. Set $I_{j}=$ $\left(u_{1}, \ldots, u_{j}\right)$ and $L_{j}=\left(u_{1}, \ldots, u_{j}\right): u_{j+1}$, then, since $I_{j+1} / I_{j} \cong R / L_{j}$, we get the exact sequences

$$
0 \longrightarrow R / L_{j} \longrightarrow R / I_{j} \longrightarrow R / I_{j+1} \longrightarrow 0 .
$$

The homomorphism $R / L_{j+1} \rightarrow R / I_{j}$ is multiplication with $u_{j+1}$. Let $F^{(j)}$ be a graded free resolution of $R / I_{j}, K^{(j)}$ the Koszul complex for the regular sequence $x_{k_{1}}, \ldots, x_{k_{l}}$ with $k_{i} \in \operatorname{set}\left(u_{j+1}\right)$, and $\psi^{(j)}: K^{(j)} \rightarrow F^{(j)}$ a graded complex homomorphism lifting $R / L_{j} \rightarrow R / I_{j}$. Then the mapping cone $C\left(\psi^{(j)}\right)$ of $\psi^{(j)}$ yields a free resolution of $R / I_{j+1}$. Thus by iterated mapping cones we obtain step by step a graded free resolution of $R / I$.

Lemma 1.5. Suppose $\operatorname{deg} u_{1} \leqslant \operatorname{deg} u_{2} \leqslant \cdots \leqslant \operatorname{deg} u_{m}$. Then the iterated mapping cone $F$, derived from the sequence $u_{1}, \ldots, u_{m}$, is a minimal graded free resolution of $R / I$, and for all $i>0$, the symbols

$$
f(\sigma ; u) \quad \text { with } \quad u \in G(I), \quad \sigma \subset \operatorname{set}(u), \quad|\sigma|=i-1
$$

form a homogeneous basis of the R-module $F_{i}$. Moreover, $\operatorname{deg} f(\sigma ; u)=|\sigma|+\operatorname{deg} u$. 
We remark that if $I$ has linear quotients with respect to $u_{1}, \ldots, u_{m}$, then this does not necessarily imply that $\operatorname{deg} u_{1} \leqslant \operatorname{deg} u_{2} \leqslant \cdots \leqslant \operatorname{deg} u_{m}$. In fact, $I=$ $\left(x_{1} x_{2}, x_{2} x_{3} x_{4}, x_{1} x_{3}\right)$ has linear quotients for the given order of the generators.

Proof of Lemma 1.5. We prove by induction on $j$ that $F^{(j)}$ is a minimal free resolution of $R / I_{j}$, and that $F^{(j)}$ has a basis as asserted. For $j=1$, the assertion is trivial. In homological degree $i-1$ the Koszul complex $K^{(j)}$ has the $R$-basis $e_{\sigma}=e_{j_{1}} \wedge \ldots \wedge e_{j_{i-1}}$, where $\sigma=\left\{j_{1}<j_{2}<\cdots<j_{i-1}\right\} \subset \operatorname{set}\left(u_{j+1}\right)$. Since $F_{i}^{(j+1)}=F_{i}^{(j)} \oplus K_{i-1}^{(j)}$, we obtain the desired basis from the induction hypothesis if we identify the elements $e_{\sigma}$ with $f\left(\sigma ; u_{j+1}\right)$.

In order to show that $F^{(j+1)}$ is a minimal free resolution, it suffices to show that $\operatorname{Im}\left(\psi^{(j)}\right) \subset \mathfrak{m} F^{(j)}$. Let $f\left(\sigma ; u_{j+1}\right) \in K_{i-1}^{(j)}$ and $\psi^{(j)}\left(f\left(\sigma ; u_{j+1}\right)\right)=$ $\sum_{i=1}^{j} \sum_{\tau} a_{\tau, i} f\left(\tau ; u_{i}\right)$. Since $|\tau|=|\sigma|-1$ and $\operatorname{deg} u_{j+1} \geqslant \operatorname{deg} u_{i}$ for all $i=1, \ldots, j$ it follows that $\operatorname{deg} f\left(\sigma ; u_{j+1}\right)>\operatorname{deg} f\left(\tau ; u_{i}\right)$ for all $\tau$ and $i$, and so $\operatorname{deg} a_{\tau, i}>0$ for all $\tau$ and $i$.

Corollary 1.6. The bigraded Poincaré series of an ideal with linear quotients is given by

$$
P_{R / I}(s, t)=1+\sum_{u \in G(I)}(1+s)^{|\operatorname{set}(u)|} s t^{\operatorname{deg} u} .
$$

Next we want to describe the chain maps of the graded minimal free resolution of an ideal with linear quotients as explicitly as possible. It will turn out that the maps are described similarly as in the Eliahou-Kervaire resolution [8] provided we impose some extra condition on the linear quotients.

Let $I$ have linear quotients with respect to the sequence of generators $u_{1}, \ldots, u_{m}$, and set as before $I_{j}=\left(u_{1}, \ldots, u_{j}\right)$ for $j=1, \ldots, m$. Let $M(I)$ be the set of all monomials in $I$. The map $g: M(I) \rightarrow G(I)$ is defined as follows: we set $g(u)=u_{j}$, if $j$ is the smallest number such that $u \in I_{j}$.

Lemma 1.7. (a) For all $u \in M(I)$ one has $u=g(u) c(u)$ with some complementary factor $c(u)$, and $\operatorname{set}(g(u)) \cap \operatorname{supp}(c(u))=\emptyset$.

(b) Let $u \in M(I), u=v w$ with $v \in G(I)$ and $\operatorname{set}(v) \cap \operatorname{supp}(w)=\emptyset$. Then $v=g(u)$.

Notice that any function $M(I) \rightarrow G(I)$ satisfying Lemma 1.7(a) is uniquely determined because of Lemma 1.7(b). We call $g$ the decomposition function of $I$.

Proof of Lemma 1.7. (a) Suppose $g(u)=u_{j}$. Since $u \in I_{j}$ it is a multiple of some $u_{i}$ with $i \leqslant j$. If $i<j$, then $u \in I_{i}$, a contradiction. This shows that $g(u)$ divides $u$, i.e., $u=g(u) c(u)$ for some $c(u)$. Suppose $\operatorname{set}(g(u)) \cap \operatorname{supp}(c(u)) \neq \emptyset$, and let $i \in \operatorname{set}(g(u)) \cap \operatorname{supp}(c(u))$. Then $u=\left(x_{i} u_{j}\right)\left(c(u) / x_{i}\right) \in I_{j-1}$, a contradiction.

(b) Let $u \in M(I)$. Suppose there exist $u_{i}, u_{j} \in G(I)$ such that $u=u_{i} v_{i}=u_{j} v_{j}$ for some monomials $v_{i}$ and $v_{j}$, and $\operatorname{set}\left(u_{i}\right) \cap \operatorname{supp}\left(v_{i}\right)=\emptyset=\operatorname{set}\left(u_{j}\right) \cap \operatorname{supp}\left(v_{j}\right)$. We may assume that $i<j$. Then the equation $u_{i} v_{i}=u_{j} v_{j}$ implies that $v_{j} \in$ $I_{j-1}: u_{j}$. Hence there exists $k \in \operatorname{set}\left(u_{j}\right)$ such that $x_{k} \mid v_{j}$. In other words, we have $k \in \operatorname{set}\left(u_{j}\right) \cap \operatorname{supp}\left(v_{j}\right)$, a contradiction. 
The following properties of the decomposition function will be needed later

Lemma 1.8. Let $u, v \in M(I)$. Then $g(u v)=g(u)$ if and only if

$$
\operatorname{set}(g(u)) \cap \operatorname{supp}(v)=\emptyset \text {. }
$$

Proof. Since $u=g(u) c(u)$, we have $u v=g(u) c(u) v$. Thus if $\operatorname{set}(g(u)) \cap \operatorname{supp}(v)=\emptyset$, it also follows that $\operatorname{set}(g(u)) \cap \operatorname{supp}(c(u) v)=\emptyset$. Because of the uniqueness of the decomposition function we conclude that $g(u v)=g(u)$.

Conversely, suppose that $g(u v)=g(u)$. Then $c(u) v=c(u v)$, and $\operatorname{son} \operatorname{supp}(v) \subset$ $\operatorname{supp}(c(u v))$. Hence, since $\operatorname{supp}(c(u v))$ and $\operatorname{set}(g(u v))$ are disjoint sets, $\operatorname{supp}(v)$ and $\operatorname{set}(g(u v))$ are disjoint, too. This yields the assertion, since $g(u)=g(u v)$.

Definition 1.9. We say that the decomposition function $g: M(I) \rightarrow G(I)$ is regular, if $\operatorname{set}\left(g\left(x_{s} u\right)\right) \subset \operatorname{set}(u)$ for all $s \in \operatorname{set}(u)$ and $u \in G(I)$.

Unfortunately the decomposition function for an ideal with linear quotients is not always regular. For example, consider $I=\left(x_{2} x_{4}, x_{1} x_{2}, x_{1} x_{3}\right)$. Then with respect to the given order of the generators, $I$ has linear quotients. One checks that $\operatorname{set}\left(x_{1} x_{3}\right)=$ $\{2\}$, and that $\operatorname{set}\left(g\left(x_{2}\left(x_{1} x_{3}\right)\right)\right)=\{4\}$.

On the other hand it is obvious that stable and squarefree stable ideals have regular decomposition functions with respect to the reverse degree lexicographic order. Another large class of squarefree ideals with regular decomposition function is given by

Theorem 1.10. Let $I$ be the Stanley-Reisner ideal of a matroid. Then I has a regular decomposition function.

Proof. Let $G(I)=\left\{u_{1}, \ldots, u_{n}\right\}$ with $u_{1}>\cdots>u_{n}$ in the reverse lexicographic order. We will set $u=u_{n}$ for convenience. Then

$$
\operatorname{set}(u)=\left\{i: \operatorname{supp}\left(u_{k}\right) \backslash \operatorname{supp}(u)=\{i\} \text {, for some } u_{k}>u\right\} \text {. }
$$

Take an arbitrary element $i \in \operatorname{set}(u)$, then we have

$$
\operatorname{set}\left(g\left(x_{i} u\right)\right)=\left\{j: \operatorname{supp}\left(u_{l}\right) \backslash \operatorname{supp}\left(g\left(x_{i} u\right)\right)=\{j\}, \text { for some } u_{l}>g\left(x_{i} u\right)\right\} .
$$

Now we will prove that $g$ is regular, namely that $\operatorname{set}\left(g\left(x_{i} u\right)\right) \subset \operatorname{set}(u)$.

We first note the following:

$$
g\left(x_{i} u\right)=\frac{x_{i} u}{x_{j(i)}} \quad \text { for some } \quad j(i) \in \operatorname{supp}(u), \quad i<j(i),
$$

and for arbitrary $j \in \operatorname{set}\left(g\left(x_{i} u\right)\right)$,

$$
\{j\}=\operatorname{supp}\left(u_{l}\right) \backslash((\operatorname{supp}(u) \cup\{i\}) \backslash j(i)) .
$$

Notice that, since $i \in \operatorname{supp}\left(g\left(x_{i} u\right)\right)$ by $(2)$, we have $i \neq j$. In the following, we will prove that $j \in \operatorname{set}(u)$.

Now we first consider the case of $i \notin \operatorname{supp}\left(u_{l}\right)$. From $(2)$, we have

$$
\{j\}=\operatorname{supp}\left(u_{l}\right) \backslash(\operatorname{supp}(u) \backslash j(i)) .
$$


Now assume that $j(i) \in \operatorname{supp}\left(u_{l}\right)$. Then we must have $j=j(i)$, and $\operatorname{supp}\left(u_{l}\right)=$ $\operatorname{supp}(u)$, a contradiction. Thus we have $j(i) \notin \operatorname{supp}\left(u_{l}\right)$ and

$$
\{j\}=\operatorname{supp}\left(u_{l}\right) \backslash \operatorname{supp}(u) .
$$

Since $u_{l}>g\left(x_{i} u\right)>u$, this means that $j \in \operatorname{set}(u)$.

Next we consider the case of $i \in \operatorname{supp}\left(u_{l}\right)$. From (2), we have

$$
\{i, j\}=\operatorname{supp}\left(u_{l}\right) \backslash(\operatorname{supp}(u) \backslash j(i)) .
$$

Now we have $j(i) \notin \operatorname{supp}\left(u_{l}\right)$. In fact, assume that $j(i) \in \operatorname{supp}\left(u_{l}\right)$. Then we have $j(i) \in\{i, j\}$ by (3). Moreover, $j(i)=j$ since $i<j(i)$. But then we have $x_{i} u=$ $x_{j(i)} g\left(x_{i} u\right)=x_{j} g\left(x_{i} u\right)$ by (1). Applying the decomposition function $g$, we obtain $g\left(x_{i} u\right)=g\left(x_{j} g\left(x_{i} u\right)\right)$. This contradicts the assumption $j \in \operatorname{set}\left(g\left(x_{i} u\right)\right)$. Thus we have

$$
\{i, j\}=\operatorname{supp}\left(u_{l}\right) \backslash \operatorname{supp}(u) .
$$

Since $\left\{\operatorname{supp}\left(u_{i}\right)\right\}_{i}$ is a basis of a matroid, there exists some $k \in \operatorname{supp}(u) \backslash \operatorname{supp}\left(u_{l}\right)$ such that $\left(x_{k} / x_{i}\right) u_{l} \in G(I)$. We denote this element by $u_{p}$. Moreover we have

$$
\begin{aligned}
\{j\} & =\operatorname{supp}\left(u_{l}\right) \backslash(\operatorname{supp}(u) \cup\{i\} \backslash j(i)) \\
& =\operatorname{supp}\left(u_{l}\right) \backslash(\operatorname{supp}(u) \cup\{i\}) \\
& =\left(\operatorname{supp}\left(u_{l}\right) \backslash i\right) \backslash \operatorname{supp}(u) \\
& =\left(\operatorname{supp}\left(u_{l}\right) \backslash i\right) \cup\{k\} \backslash \operatorname{supp}(u) \\
& =\operatorname{supp}\left(u_{p}\right) \backslash \operatorname{supp}(u) .
\end{aligned}
$$

If $u_{p}>u$, this equation means $j \in \operatorname{set}(u)$, and we are done.

Now in the rest of the proof, we assume $u_{p}<u$. Then, since $u_{p}=\left(x_{k} / x_{i}\right) u_{l}$ and $u_{l}>u$, we must have $i<k$. Assume that $k=j(i)$. Since all bases of a matroid have the same cardinality and $\left|\operatorname{supp}\left(u_{l}\right) \backslash \operatorname{supp}(u)\right|=2$ by (4), there must be some element $\zeta \in \operatorname{supp}(u)$ such that

$$
\{\zeta, j(i)\}=\operatorname{supp}(u) \backslash \operatorname{supp}\left(u_{l}\right) .
$$

Then we have

$$
u_{l}=\frac{x_{i} x_{j} u}{x_{k} x_{\zeta}}, \quad u_{p}=\frac{x_{k} u_{l}}{x_{i}}=\frac{x_{j} u}{x_{\zeta}}
$$

and $u_{p}<u$ implies $j>\zeta$. Moreover, $g\left(x_{i} u\right)=\left(x_{i} / x_{j(i)}\right) u=\left(x_{i} / x_{k}\right) u$, by (1). Thus we have $u_{l}=\left(x_{j} / x_{\zeta}\right) g\left(x_{i} u\right)$ and $u_{l}<g\left(x_{i} u\right)$. But this contradicts with the assumption that $u_{l}>g\left(x_{i} u\right)$. Consequently, we must have $k \neq j(i)$.

Now we have

$$
\{k, j(i)\}=\operatorname{supp}(u) \backslash \operatorname{supp}\left(u_{l}\right) .
$$

Since

$$
u_{l}=\frac{x_{j} x_{i} u}{x_{k} x_{j(i)}}>g\left(x_{i} u\right)=\frac{x_{i} u}{x_{j(i)}},
$$

we have $j<k$. Since moreover we have $i<j(i)$ and $i<k$, there are five possibilities of total order on $i, j, k$ and $j(i)$ left, namely (i) $i<j<k<j(i)$, (ii) $i<j<j(i)<k$, (iii) $j<i<k<j(i)$, (iv) $j<i<j(i)<k$, or (v) $i<j(i)<j<k$. It is easy to 
check that only in the last case one has $u_{p}<u$. So we assume $i<j(i)<j<k$ in the following.

Since $\left\{\operatorname{supp}\left(u_{i}\right)\right\}_{i}$ is the basis of a matroid, we have, for $k$, either $\left(x_{i} / x_{k}\right) u \in G(I)$ or $\left(x_{j} / x_{k}\right) u \in G(I)$, by (4). If $\left(x_{j} / x_{k}\right) u \in G(I)$, then

$$
\{j\}=\operatorname{supp}\left(\frac{x_{j} u}{x_{k}}\right) \backslash \operatorname{supp}(u),
$$

and, since $j<k$, we get $\left(u_{j} / x_{k}\right) u>u$. Hence we have $j \in \operatorname{set}(u)$. Now assume that $\left(x_{j} / x_{k}\right) u \notin G(I)$. Then we must have $\left(x_{i} / x_{k}\right) u \in G(I)$. Since $i<k$, we get $\left(x_{i} / x_{k}\right) u>u$. But $g\left(x_{i} u\right)=\left(x_{i} / x_{j(i)}\right) u<\left(x_{i} / x_{k}\right) u$ since $j(i)<k$, a contradiction. Hence this case does not happen.

Lemma 1.11. If $g: M(I) \rightarrow G(I)$ is a regular decomposition function, then

$$
g\left(x_{s} g\left(x_{t} u\right)\right)=g\left(x_{t} g\left(x_{s} u\right)\right) \quad \text { for all } u \in M(I) \quad \text { and all } \quad s, t \in \operatorname{set}(u) \text {. }
$$

Proof. If $s \notin \operatorname{set}\left(g\left(x_{t} u\right)\right)$, then Lemma 1.8 implies that $g\left(x_{s} g\left(x_{t} u\right)\right)=g\left(g\left(x_{t} u\right)\right)=$ $g\left(x_{t} u\right)$, and if $s \in \operatorname{set}\left(g\left(x_{t} u\right)\right)$, then $\operatorname{set}\left(g\left(x_{s} g\left(x_{t} u\right)\right)\right) \subset \operatorname{set}\left(g\left(x_{t} u\right)\right)$ since $g$ is regular. Thus in any case, it follows that $\operatorname{set}\left(g\left(x_{s} g\left(x_{t} u\right)\right)\right) \subset \operatorname{set}\left(g\left(x_{t} u\right)\right)$. Hence

$$
\operatorname{set}\left(g\left(x_{s} g\left(x_{t} u\right)\right)\right) \cap \operatorname{supp}\left(c\left(x_{t} u\right)\right)=\emptyset,
$$

so that by Lemma 1.8 again we have $g\left(x_{s} g\left(x_{t} u\right) c\left(x_{t} u\right)\right)=g\left(x_{s} g\left(x_{t} u\right)\right)$. Therefore, by the uniqueness of the decomposition function (see Lemma 1.7(b)), the equation $x_{s} x_{t} u=\left(x_{s} g\left(x_{t} u\right)\right) c\left(x_{t} u\right)$ yields $g\left(x_{s} g\left(x_{t} u\right)\right)=g\left(x_{s} x_{t} u\right)$. This implies the assertion.

The exchange property of the decomposition function in Lemma 1.11 is weaker than the regularity, as is demonstrated by the following example: The ideal $I=$ $\left(x_{1} x_{3}, x_{2} x_{3}, x_{1} x_{5}, x_{3} x_{4}, x_{4} x_{5}\right)$ has linear quotients with respect to the given order of the generators. One checks that the exchange property holds. But $\operatorname{since} \operatorname{set}\left(x_{4} x_{5}\right)=$ $\{1,3\}$, and $\operatorname{set}\left(g\left(x_{3}\left(x_{4} x_{5}\right)\right)\right)=\{1,2\}, g$ is not regular.

The following theorem is the main result of this section. It generalizes the theorem of Eliahou-Kervaire ([8]) for stable ideals and that of Aramova-Herzog-Hibi ([2]) for squarefree stable ideals. For convenience, and to avoid unnecessary distinctions, we extend the definition introduced in Lemma 1.5 and set $f(\sigma ; u)=0$ if $\sigma \not \subset \operatorname{set}(u)$.

Theorem 1.12. Let $I$ be a monomial ideal with linear quotients, and $F$ the graded minimal free resolution of $R / I$. Suppose the decomposition function $g: M(I) \rightarrow$ $G(I)$ is regular. Then the chain map $\partial$ of $F$ is given by

$$
\partial(f(\sigma ; u))=-\sum_{t \in \sigma}(-1)^{\alpha(\sigma ; t)} x_{t} f(\sigma \backslash t ; u)+\sum_{t \in \sigma}(-1)^{\alpha(\sigma ; t)} \frac{x_{t} u}{g\left(x_{t} u\right)} f\left(\sigma \backslash t ; g\left(x_{t} u\right)\right),
$$

if $\sigma \neq \emptyset$, and

$$
\partial(f(\emptyset ; u))=u \quad \text { otherwise } .
$$

Here $\alpha(\sigma ; t)=|\{s \in \sigma: s<t\}|$.

Proof. Let $I$ have linear quotients with respect to the sequence $u_{1}, \ldots, u_{m}$. We show by induction on $j$, that $F^{(j)}$ has the desired chain map. For $j=1$, the assertion is 
trivial. Since $F^{(j+1)}$ is the mapping cone of $\psi^{(j)}: K^{(j)} \rightarrow F^{(j)}$ it follows that $F^{(j)}$ is a subcomplex of $F^{(j+1)}$ and it suffices to check the formula for the chain map on the basis elements $f\left(\sigma ; u_{j+1}\right)$. By the definition of the mapping cone of $\psi^{(j)}$ we have $\partial\left(f\left(\sigma ; u_{j+1}\right)\right)=-\partial_{1}\left(f\left(\sigma ; u_{j+1}\right)\right)+\psi^{(j)}\left(f\left(\sigma ; u_{j+1}\right)\right)$, where $\partial_{1}$ is the chain map of the Koszul complex $K^{(j)}$. Thus in order to prove the asserted formula it remains to show that we can define $\psi^{(j)}$ as

$$
\psi^{(j)}\left(f\left(\sigma ; u_{j+1}\right)\right)=\sum_{t \in \sigma}(-1)^{\alpha(\sigma ; t)} \frac{x_{t} u_{j+1}}{g\left(x_{t} u_{j+1}\right)} f\left(\sigma \backslash t ; g\left(x_{t} u_{j+1}\right)\right),
$$

if $\sigma \neq \emptyset$, and $\psi^{(j)}\left(f\left(\emptyset ; u_{j+1}\right)\right)=u_{j+1}$, otherwise.

To verify this we must prove that $\psi^{(j)} \circ \partial_{1}=\partial \circ \psi^{(j)}$. In order to simplify notation we set $u=u_{j+1}$ and $\psi=\psi^{(j)}$. Then for $t \in \operatorname{set}(u)$ we have

$$
\begin{aligned}
\left(\psi \circ \partial_{1}\right)(f(\{t\} ; u)) & =\psi\left(x_{t} f(\emptyset ; u)\right) \\
& =x_{t} u,
\end{aligned}
$$

while on the other hand

$$
\begin{aligned}
(\partial \circ \psi)(f(\{t\} ; u)) & =\partial\left(\frac{x_{t} u}{g\left(x_{t} u\right)} f\left(\emptyset ; g\left(x_{t} u\right)\right)\right. \\
=\frac{x_{t} u}{g\left(x_{t} u\right)} g\left(x_{t} u\right) & =x_{1} u .
\end{aligned}
$$

Now let $\sigma \subset \operatorname{set}(u)$ with $|\sigma| \geqslant 2$. Then

$$
\begin{aligned}
\left(\psi \circ \partial_{1}\right)(f(\sigma ; u)) & =\sum_{t \in \sigma}(-1)^{\alpha(\sigma ; t)} x_{t} \psi(f(\sigma \backslash t ; u)) \\
& =\sum_{t \in \sigma}(-1)^{\alpha(\sigma ; t)} x_{t}\left(\sum_{s \in \sigma \backslash t}(-1)^{\alpha(\sigma \backslash t ; s)} \frac{x_{s} u}{g\left(x_{s} u\right)} f\left(\sigma \backslash\{s, t\} ; g\left(x_{s} u\right)\right)\right. \\
& =\sum_{t \in \sigma} \sum_{\substack{s \in \sigma \backslash t \\
s<t}}(-1)^{\alpha(\sigma ; t)+\alpha(\sigma ; s)} \frac{x_{t} x_{s} u}{g\left(x_{s} u\right)} f\left(\sigma \backslash\{s, t\} ; g\left(x_{s} u\right)\right) \\
& -\sum_{t \in \sigma} \sum_{\substack{s \in \sigma \backslash t \\
s>t}}(-1)^{\alpha(\sigma ; t)+\alpha(\sigma ; s)} \frac{x_{t} x_{s} u}{g\left(x_{s} u\right)} f\left(\sigma \backslash\{s, t\} ; g\left(x_{s} u\right)\right) .
\end{aligned}
$$

Exchanging the role of $s$ and $t$ in the second sum, we obtain

$$
\begin{aligned}
\left(\psi \circ \partial_{1}\right)(f(\sigma ; u)) & =\sum_{t \in \sigma} \sum_{\substack{s \in \sigma \backslash t \\
s<t}}(-1)^{\alpha(\sigma ; t)+\alpha(\sigma ; s)} \frac{x_{t} x_{s} u}{g\left(x_{s} u\right)} f\left(\sigma \backslash\{s, t\} ; g\left(x_{s} u\right)\right) \\
& -\sum_{s \in \sigma} \sum_{\substack{t \in \sigma \backslash s \\
s<t}}(-1)^{\alpha(\sigma ; t)+\alpha(\sigma ; s)} \frac{x_{t} x_{s} u}{g\left(x_{t} u\right)} f\left(\sigma \backslash\{s, t\} ; g\left(x_{t} u\right)\right) .
\end{aligned}
$$

On the other hand we have

$$
(\partial \circ \psi)(f(\sigma ; u))=\sum_{t \in \sigma}(-1)^{\alpha(\sigma ; t)} \frac{x_{t} u}{g\left(x_{t} u\right)} \partial\left(f\left(\sigma \backslash t ; g\left(x_{t} u\right)\right),\right.
$$


and

$$
\begin{aligned}
\partial\left(f\left(\sigma \backslash t ; g\left(x_{t} u\right)\right)\right)= & -\sum_{s \in \sigma \backslash t}(-1)^{\alpha(\sigma ; s)} x_{s} f\left(\sigma \backslash\{s, t\} ; g\left(x_{t} u\right)\right) \\
& +\sum_{s \in \sigma \backslash t}(-1)^{\alpha(\sigma s)} \frac{x_{s} g\left(x_{t} u\right)}{g\left(x_{s} g\left(x_{t} u\right)\right)} f\left(\sigma \backslash\{s, t\} ; g\left(x_{s} g\left(x_{t} u\right)\right)\right.
\end{aligned}
$$

Before we continue our calculation we notice that it may happen that $\sigma \backslash\{t\} \not \subset$ $\operatorname{set}\left(g\left(x_{t} u\right)\right)$, in which case $f\left(\sigma \backslash\{t\} ; g\left(x_{t} u\right)\right)=0$, by convention. Thus the right hand side of the equation should also be zero.

In fact, let $s \in \sigma \backslash\{t\}$. If $\sigma \backslash\{s, t\} \not \subset \operatorname{set}\left(g\left(x_{t} u\right)\right)$, then $\sigma \backslash\{s, t\} \not \subset \operatorname{set}\left(g\left(x_{s} g\left(x_{t} u\right)\right)\right)$, since $g$ is regular, and so the corresponding summands are zero. Otherwise, $\sigma \backslash$ $\{s, t\} \subset \operatorname{set}\left(g\left(x_{t} u\right)\right)$. But then $s \notin \operatorname{set}\left(g\left(x_{t} u\right)\right)$, so that $g\left(x_{s}\left(g\left(x_{t} u\right)\right)\right)=g\left(x_{t} u\right)$, by Lemma 1.8. Therefore,

$$
\frac{x_{s} g\left(x_{t} u\right)}{g\left(x_{s} g\left(x_{t} u\right)\right)} f\left(\sigma \backslash\{s, t\} ; g\left(x_{s} g\left(x_{t} u\right)\right)\right)=x_{s} f\left(\sigma \backslash\{s, t\} ; g\left(x_{t} u\right)\right) .
$$

Hence we see that the summands on the right hand side of the equation are either zero or cancel each other, as we wanted to show.

Now continuing with our calculation we get

$$
\begin{aligned}
\partial\left(f\left(\sigma \backslash t ; g\left(x_{t} u\right)\right)\right)= & -\sum_{\substack{s \in \sigma \backslash t \\
s<t}}(-1)^{\alpha(\sigma ; s)} x_{s} f\left(\sigma \backslash\{s, t\} ; g\left(x_{t} u\right)\right) \\
& +\sum_{\substack{s \in \sigma \backslash t \\
s>t}}(-1)^{\alpha(\sigma ; s)} x_{s} f\left(\sigma \backslash\{s, t\} ; g\left(x_{t} u\right)\right) \\
& +\sum_{\substack{s \in \sigma \backslash t \\
s<t}}(-1)^{\alpha(\sigma ; s)} \frac{x_{s} g\left(x_{t} u\right)}{g\left(x_{s} g\left(x_{t} u\right)\right)} f\left(\sigma \backslash\{s, t\} ; g\left(x_{s} g\left(x_{t} u\right)\right)\right) \\
& -\sum_{\substack{s \in \sigma \backslash t \\
s>t}}(-1)^{\alpha(\sigma ; s)} \frac{x_{s} g\left(x_{t} u\right)}{g\left(x_{s} g\left(x_{t} u\right)\right)} f\left(\sigma \backslash\{s, t\} ; g\left(x_{s} g\left(x_{t} u\right)\right)\right) .
\end{aligned}
$$

Exchanging the role of $s$ and $t$ in the second and fourth sum, and substituting into (7) we obtain

$$
\begin{aligned}
(\partial \circ \psi)(f(\sigma ; u))= & -\sum_{t \in \sigma} \sum_{\substack{s \in \sigma \backslash t \\
s<t}}(-1)^{\alpha(\sigma ; t)+\alpha(\sigma ; s)} \frac{x_{s} x_{t} u}{g\left(x_{t} u\right)} f\left(\sigma \backslash\{s, t\} ; g\left(x_{t} u\right)\right) \\
& +\sum_{s \in \sigma} \sum_{\substack{t \in \sigma \backslash s \\
s<t}}(-1)^{\alpha(\sigma ; t)+\alpha(\sigma ; s)} \frac{x_{s} x_{t} u}{g\left(x_{s} u\right)} f\left(\sigma \backslash\{s, t\} ; g\left(x_{s} u\right)\right) \\
& +\sum_{t \in \sigma} \sum_{\substack{s \in \sigma \backslash t \\
s<t}}(-1)^{\alpha(\sigma ; s)+\alpha(\sigma ; t)} \frac{x_{s} x_{t} u}{g\left(x_{s} g\left(x_{t} u\right)\right)} f\left(\sigma \backslash\{s, t\} ; g\left(x_{s} g\left(x_{t} u\right)\right)\right) \\
& -\sum_{s \in \sigma} \sum_{\substack{t \in \sigma \backslash s \\
s<t}}(-1)^{\alpha(\sigma ; s)+\alpha(\sigma ; t)} \frac{x_{s} x_{t} u}{g\left(x_{t} g\left(x_{s} u\right)\right)} f\left(\sigma \backslash\{s, t\} ; g\left(x_{t} g\left(x_{s} u\right)\right)\right) .
\end{aligned}
$$


The last two double sums in this expression cancel each other since by Lemma 1.11 we have $g\left(x_{s} g\left(x_{t} u\right)\right)=g\left(x_{t} g\left(x_{s} u\right)\right)$ for all $s, t \in \operatorname{set}(u)$. Hence a comparison with (6) yields the conclusion.

\section{DG algebra structures on trivial extensions}

In this section we describe constructions which in some cases allow to define algebra structures on free resolutions.

We shall need the following concepts: Let $R$ be a commutative ring with a unit. $D G$ algebra $A$ is a complex $(A, \partial)$ of $R$-modules with $A_{i}=0$ for $i<0$, which admits the structure of a unitary, associative, graded commutative algebra such that the Leibniz rule is satisfied:

$$
\partial(a b)=\partial(a) b+(-1)^{|a|} a \partial(b) \text { for all homogeneous elements } \quad a, b \in A .
$$

Here $|a|$ denotes the degree of $a$.

Let $I \subset R$ be an ideal. A $D G$ algebra resolution of $R / I$ is a DG algebra $A$ which is a $R$-free resolution of $R / I$.

A two-sided $D G$ module $M$ over $A$ is a complex of $R$-modules together with complex homomorphism $A \otimes_{R} M \rightarrow M, a \otimes m \mapsto a m$ and $M \otimes_{R} A \rightarrow M, m \otimes a \mapsto$ $m a$, which are unitary and associative, satisfy the Leibniz rule, and the rules:

$$
(a m) b=a(m b) \quad \text { and } \quad a m=(-1)^{|a||m|} m a,
$$

for all homogeneous elements $a, b \in A$ and $m \in M$. We refer the reader to [1] for details.

The following lemma which, in a different context, can be found in [12] and which mimics for DG algebras the trivial extension of an algebra by a module (the so-called Nagata extension), is the basis of our theory.

Lemma 2.1. Let $A$ be a $D G$ algebra, $M$ a two-sided $D G A$-module and $\psi: M \rightarrow A$ a DG module homomorphism. Suppose $\psi$ satisfies the condition:

$$
\psi(m) n=m \psi(n), \quad \text { for all } m, n \in M .
$$

Then the mapping cone $C(\psi)$ of $\psi$ has a DG algebra structure with Nagata product

$$
(a, m)(b, n)=\left(a b,(-1)^{|a|} a n+m b\right),
$$

for all $(a, m),(b, n) \in C(\psi)$.

The mapping cone with the DG algebra structure as in defined in Lemma 2.1 will be denoted by $A * M$.

Let $I=\left(f_{1}, \ldots, f_{n+1}\right) \subset R$ be an ideal. Set $J=\left(f_{1}, \ldots, f_{n}\right)$, and let $L=$ $\left(f_{1}, \ldots, f_{n}\right): f_{n+1}$. Let $A$ be a free $R$-resolution of $R / J, M$ a free $R$-resolution of $R / L$, and $\psi: M \rightarrow A$ be a complex homomorphism extending $R / L \rightarrow R / J$, and $C(\psi)$ a mapping cone.

Assume that $A$ and $M$ are DG algebra resolutions of $R / J$ and $R / L$, respectively. We want to give to $C(\psi)$ a DG-algebra structure by applying Lemma 2.1. To this end, we must define a suitable action of $A$ on $M$, and the complex homomorphism 
$\psi$ has to be chosen such that it is a DG module homomorphism over $A$ satisfying the condition of Lemma 2.1.

We first define an action of $A$ on $M$ : Since $J \subset L$, there is a natural surjection $R / J \rightarrow R / L$, which induces a complex homomorphism $\varphi: A \rightarrow M$. Assume that $\varphi$ can be chosen to be a DG-algebra homomorphism. Then the action of $A$ on $M$ will be defined by:

$$
a m=\varphi(a) m \text { and } m a=m \varphi(a) \text { for all } a \in A \text { and all } m \in M,
$$

where the product on the right hand side of the equation is multiplication in the DG-algebra $M$. It is clear that with this action $M$ is a two-sided DG $A$-module over A.

Lemma 2.2. (a) Let $\psi: M \rightarrow A$ be complex homomorphism such that $\varphi \circ \psi=$ $f_{n+1} \mathrm{id}_{M}$. Assume that either

(i) $f_{n+1}$ is a non-zerodivisor of $R$, and $\operatorname{Im} \psi$ is an ideal of $A$, or

(ii) $\varphi$ is injective.

Then $\psi$ is a $D G$ A-module homomorphism.

(b) Let $\psi: M \rightarrow A$ be a $D G A$-module homomorphism. The following conditions are equivalent:

(i) $\psi$ satisfies the condition of Lemma 2.1,

(ii) $\varphi \circ \psi=f_{n+1} \operatorname{id}_{M}$.

Proof. (a) In order to prove that $\psi$ a a DG module homomorphism, we must show that

$$
\psi(\varphi(a) m)=a \psi(m), \quad \text { for all } \quad a \in A \text { and } m \in M \text {. }
$$

Assuming case (i), there exists $n \in M$ with $a \psi(m)=\psi(n)$. Also, since $\varphi$ is a DG-algebra homomorphism, we have $\varphi(a \psi(m))=\varphi(a) \varphi(\psi(m))=f_{n+1} \varphi(a) m$ by $\varphi \circ \psi=f_{n+1} \operatorname{id}_{M}$. Therefore,

$$
f_{n+1} \varphi(a) m=\varphi(a \psi(m))=\varphi(\psi(n))=f_{n+1} n,
$$

so that $n=\varphi(a) m$. Applying $\psi$ we obtain the desired equation.

In case (ii) the assertion follows again, since

$$
\begin{aligned}
\varphi(\psi(\varphi(a) m)-a \psi(m))) & =\varphi(\psi(\varphi(a) m))-\varphi(a \psi(m)) \\
& =f_{n+1} \varphi(a) m-\varphi(a) \varphi(\psi(m)) \\
& =f_{n+1} \varphi(a) m-\varphi(a)\left(f_{n+1} m\right) \\
& =0 .
\end{aligned}
$$

(b) Suppose $\psi$ satisfies the condition of Lemma 2.1, then

$$
\varphi(\psi(m)))=\psi(m) 1_{M}=m \psi\left(1_{M}\right)=m f_{n+1}=f_{n+1} m
$$

for all $m \in M$. Hence we have $\psi \circ \varphi=f_{n+1} \mathrm{id}_{M}$. Conversely, if (ii) is satified, then for all $m, n \in M$ we have

$$
\psi(m) n=(\varphi \circ \psi)(m) n=f_{n+1} m n=m\left(f_{n+1} n\right)=m(\varphi \circ \psi)(n)=m \psi(n) .
$$




\section{Koszul type resolutions}

We introduce the following notion.

Definition 3.1. Let $I \subset R$ be an ideal. $A D G$ algebra resolution $A$ of $R / I$ over $R$ is of Koszul type (of length $n$ ), if for all $i$

(i) $\operatorname{rank} A_{i}=\left(\begin{array}{c}n \\ i\end{array}\right)$,

(ii) the homomorphism $A_{i} \rightarrow \operatorname{Hom}_{R}\left(A_{n-i}, A_{n}\right)$ which assigns to each $a \in A_{i}$ the map $\alpha_{a}: A_{n-i} \rightarrow A_{n}$ with $\alpha_{a}(b)=a b$ is injective.

Let, as in Section 2, $A$ and $M$ be DG algebra resolutions of $R / J$ and $R / I$, respectively, and let $\varphi: A \rightarrow M$ be a DG algebra homomorphism.

Lemma 3.2. Assume that $R$ is a domain with quotient field $Q$, and that $A$ and $M$ are of Koszul type of length $n$. Fix a basis e for $A_{n}$ and a basis $\bar{e}$ for $M_{n}$, and assume that $\varphi(e)=\delta \bar{e}$ with $\delta \neq 0$. Then there is a unique complex homomorphism $\tilde{\varphi}: M \otimes_{R} Q \rightarrow A \otimes_{R} Q$ satisfying

$$
\tilde{\varphi}(m) a=m \varphi(a),
$$

for all $0 \leqslant i \leqslant n, a \in A_{n-i}$ and $m \in M_{i}$, where the equation means equality between the coefficients of the bases e and $\bar{e}$. Moreover, $\tilde{\varphi} \circ \varphi=\delta \operatorname{id}_{A \otimes Q}$ and $\varphi \circ \tilde{\varphi}=\delta \operatorname{id}_{M \otimes Q}$.

Proof. Let $\varepsilon: M_{n} \otimes Q \rightarrow A_{n} \otimes Q$ be the isomorphism with $\varepsilon(\bar{e})=e$. Given $m \in$ $M_{i} \otimes Q$, we define the $Q$-linear map $\gamma: A_{n-i} \otimes Q \rightarrow A_{n} \otimes Q$ by $\gamma(a)=\varepsilon(m \varphi(a))$. (Here we write $\varphi$ instead of $\varphi \otimes Q$, in order to simplify notation.)

Since by assumption the natural map $A_{i} \rightarrow \operatorname{Hom}_{R}\left(A_{n-i}, A_{n}\right)$ is injective, the induced map $A_{i} \otimes Q \rightarrow \operatorname{Hom}_{Q}\left(A_{n-i} \otimes Q, A_{n} \otimes Q\right)$ is again injective, and hence must even be bijective since it is a linear map of vector spaces of equal dimension. Therefore, there exists $b \in A_{i} \otimes Q$ with $b a=\gamma(a)=m \varphi(a)$ for all $a \in A_{n-i}$, and we set $\tilde{\varphi}(m)=b$. Then $\tilde{\varphi}(m) a=m \varphi(a)$.

For arbitrary elements $m \in M_{i+1} \otimes Q$ and $a \in A_{n-i} \otimes Q$, we have

$$
\tilde{\varphi}(\partial(m)) a=\partial(m) \varphi(a) .
$$

Since $m \varphi(a) \in M_{n+1} \otimes Q=0$, we have $0=\partial(m \varphi(a))=\partial(m) \varphi(a)+(-1)^{|m|} m \partial(\varphi(a))$. Hence

$$
\begin{aligned}
\partial(m) \varphi(a) & =-(-1)^{|m|} m \partial(\varphi(a)) \\
& =-(-1)^{|m|} m \varphi(\partial(a)) \\
& =-(-1)^{|m|} \tilde{\varphi}(m) \partial(a),
\end{aligned}
$$

and since $\tilde{\varphi}(m) a \in A_{n+1}=0$, we have

$$
0=\partial(\tilde{\varphi}(m) a)=\partial(\tilde{\varphi}(m)) a+(-1)^{|\tilde{\varphi}(m)|} \tilde{\varphi}(m) \partial(a),
$$

so that

$$
\tilde{\varphi}(m) \partial(a)=(-1)^{|m|+1} \partial(\tilde{\varphi}(m)) a
$$

Consequently,

$$
\tilde{\varphi}(\partial(m)) a=\partial(\tilde{\varphi}(m)) a
$$


for all $a \in A_{n-i} \otimes Q$ and all $m \in M_{i+1} \otimes Q$. Therefore, since $A$ is of Koszul type we conclude that

$$
\tilde{\varphi}(\partial(m))=\partial(\tilde{\varphi}(m))
$$

which shows that $\tilde{\varphi}$ is indeed a complex homomorphism.

Let $a \in A_{i} \otimes Q$ and $b \in A_{n-i} \otimes Q$. Then $\tilde{\varphi}(\varphi(a)) b=\varphi(a) \varphi(b)=\varphi(a b)=\delta a b$. Hence since $A$ is of Koszul type, it follows that $\tilde{\varphi}(\varphi(a))=\delta a$. In other words, $\tilde{\varphi} \circ \varphi=\delta \mathrm{id}_{A \otimes Q}$. This implies in particular that $\varphi: A_{i} \otimes Q \rightarrow M_{i} \otimes Q$ is injective. However, since $A_{i} \otimes Q$ and $M_{i} \otimes Q$ have the same $Q$-dimension, we see that $\varphi$ is an isomorphism with $\varphi^{-1}=\delta^{-1} \tilde{\varphi}$. Therefore, $\varphi \circ \delta^{-1} \tilde{\varphi}=\operatorname{id}_{M_{i} \otimes Q}$, and so $\varphi \circ \tilde{\varphi}=$ $\delta \operatorname{id}_{M_{i} \otimes Q}$, as desired.

Now Lemma 2.2 and Lemma 3.2 imply immediately

Corollary 3.3. With the assumptions and the notation of Lemma 3.2, suppose that $f_{n+1} \tilde{\varphi}(M) \subset \delta A$, and set $\psi=\left(f_{n+1} / \delta\right) \tilde{\varphi}$. If $M$ is viewed a two-sided A-module via $\varphi$, then $\psi: M \rightarrow A$ is a DG module homomorphism satisfying the condition of Lemma 2.1. In particular, $A * M$ is defined, and $A * M$ is of Koszul type of length $n+1$.

Proof. It remains to be shown that $A * M$ is of Koszul type of length $n+1$. In fact, we have $(A * M)_{i}=A_{i} \oplus M_{i-1}$, and so $\operatorname{rank}(A * M)_{i}=\operatorname{rank} A_{i}+\operatorname{rank} M_{i-1}=$ $\left(\begin{array}{c}n \\ i\end{array}\right)+\left(\begin{array}{c}n \\ i-1\end{array}\right)=\left(\begin{array}{c}n+1 \\ i\end{array}\right)$.

Finally, let $(a, m) \in(A * M)_{i},(a, m) \neq 0$. We must show that there exists $(b, n) \in$ $(A * M)_{n+1-i}$ such that $(a, m)(b, n) \neq 0$. By the definition of our multiplication we get $(a, m)(b, n)=\left(0,(-1)^{|a|} \varphi(a) n+m \varphi(b)\right)$.

There are two cases to consider. If $a \neq 0$, we let $b=0$, and have to show that there exists $n \in M$ with $\varphi(a) n \neq 0$. Now since $M$ is of Koszul type, there exists $w \in M_{i}$ such that $w n \neq 0$. But recall that $A_{i} \otimes Q \rightarrow M_{i} \otimes Q$ is an isomorphism via $\varphi$. Thus, since $a \neq 0, \varphi(a) n \neq 0$ for arbitrary $n \neq 0$, and by the isomorphism $A_{n} \otimes Q \rightarrow M_{n} \otimes Q$ between one dimensional vector spaces we have $\lambda \in Q, \lambda \neq 0$ such that $\varphi(a) n=\lambda w n \neq 0$.

In the second case, $m \neq 0$, and we let $n=0$. Then we have to find $b \in A_{n+1-i}$ such that $m \varphi(b) \neq 0$. The rest of the argument is the same as in the first case.

Definition 3.4. A sequence $f_{1}, \ldots, f_{n}$ in $R$ is called a Koszul sequence, if for all $i=1, \ldots, n$

(i) $R /\left(f_{1}, \ldots, f_{i}\right)$ has a Koszul type resolution $A^{(i)}$ of length $i$;

(ii) $R /\left(\left(f_{1}, \ldots, f_{i-1}\right): f_{i}\right)$ has a Koszul type resolution $M^{(i-1)}$ of of length $i$;

(iii) $A^{(i)} \cong A^{(i-1)} * M^{(i-1)}$.

We will now consider some examples.

Example 3.5. Regular sequences are Koszul sequences.

Proof. Let $f_{1}, \ldots, f_{n}$ be regular sequence. For a given $i$ we let $A^{(i)}$ be t he Koszul complex for the sequence $f_{1}, \ldots, f_{i}$. Since $\left(f_{1}, \ldots, f_{i}\right): f_{i+1}=\left(f_{1}, \ldots, f_{i}\right)$, we may choose $M^{(i)}=A^{(i)}$, and $\varphi=\operatorname{id}_{A(i)}$. Then $\psi=f_{i+1} \operatorname{id}_{A^{(i)}}$. It is then easy to see that $A^{(i+1)}=A^{(i)} * A^{(i)}$ is the Koszul complex for the sequence $f_{1}, \ldots, f_{i+1}$. 
Example 3.6. Monomial sequences are Koszul sequences.

Proof. Let $f_{1}, \ldots, f_{n}$ be a sequence of monomials. The Taylor resolution $T=$ $T\left(f_{1}, \ldots, f_{n}\right)$ of this sequence admits a natural DG algebra structure, as shown by Gemeda [11] (see also [9]). As an $R$-module $T_{k}$ is the $k$ th exterior power of $T_{1}=\bigoplus_{i=1}^{n} R e_{i}$ with $R$-basis $\left\{e_{\sigma}: \sigma \subset[n],|\sigma|=k\right\}$, where $e_{\sigma}=e_{i_{1}} \wedge e_{i_{2}} \wedge \cdots \wedge e_{i_{k}}$ for $\sigma=\left\{i_{1}<i_{2}<\cdots<i_{k}\right\}$. The chain map $\partial: T_{i} \rightarrow T_{i-1}$ is defined by

$$
\partial\left(e_{\sigma}\right)=\sum_{i \in \sigma}(-1)^{\sigma(\sigma, i)} \frac{f_{\sigma}}{f_{\sigma \backslash\{i\}}} e_{\sigma \backslash\{i\}},
$$

where for $\tau \subset[n], f_{\tau}$ denotes the least common multiple of the monomials $f_{i}$ with $i \in \tau$, and where $\sigma(\sigma, i)=|\{j \in \sigma: j<i\}|$. According to Gemeda, the DG algebra structure on $T$ is given by

$$
e_{\sigma} e_{\tau}=\frac{f_{\sigma} f_{\tau}}{f_{\sigma \cup \tau}} e_{\sigma} \wedge e_{\tau}
$$

It is also known, and easy to see, that $T\left(f_{1}, \ldots, f_{n}\right)$ is obtained as the mapping cone of $\psi: T\left(g_{1}, \ldots, g_{n-1}\right) \rightarrow T\left(f_{1}, \ldots, f_{n-1}\right)$, where $g_{i}=f_{i} /\left[f_{i}, f_{n}\right]$ for $i=1, \ldots, n$, and where $\psi\left(\bar{e}_{\sigma}\right)=\left(f_{\sigma \cup\{n\}} / f_{\sigma}\right) e_{\sigma}$. Here $\left\{\bar{e}_{\sigma}: \sigma \subset[n-1]\right\}$ denotes the natural basis of $T\left(g_{1}, \ldots, g_{n-1}\right)$.

We now define an $R$-module homomorphism $\varphi: T\left(f_{1}, \ldots, f_{n}\right) \rightarrow T\left(g_{1}, \ldots, g_{n}\right)$ by

$$
\varphi\left(e_{\sigma}\right)=\frac{f_{\sigma} f_{n+1}}{f_{\sigma \cup\{n+1\}}} \bar{e}_{\sigma}, \quad \text { for all } \sigma \subset[n] .
$$

It is easy to check that $\varphi$ is an injective DG algebra homomorphism, and that $\varphi \circ \psi=$ $f_{n+1}$ id. Therefore, Lemma 2.2 implies that $\psi$ is a DG $A$-module homomorphism satisfying the condition Lemma 2.1. Thus the DG algebra $T\left(f_{1}, \ldots, f_{n}\right) * T\left(g_{1}, \ldots, g_{n}\right)$ is defined.

Consider the $R$-module homomorphism

$$
\alpha: T\left(f_{1}, \ldots, f_{n+1}\right) \longrightarrow T\left(f_{1}, \ldots, f_{n}\right) * T\left(g_{1}, \ldots, g_{n}\right)
$$

with

$$
\alpha\left(e_{\sigma}\right)= \begin{cases}\left(e_{\sigma}, 0\right), & \text { if } n+1 \notin \sigma \\ \left(0, \bar{e}_{\sigma \backslash\{n+1\}}\right), & \text { if } n+1 \in \sigma .\end{cases}
$$

We leave it to the reader to check that this DG algebra isomorphism.

It may be worthwhile to notice that with the notation of Corollary 3.3 one has $\psi=\left(f_{n+1} / \delta\right) \tilde{\varphi}$ where in this case $\delta=\left(f_{[n]} f_{n+1} / f_{[n+1]}\right)$.

Example 3.7. Let $f_{1}, \ldots, f_{n}$ and $g_{1}, \ldots, g_{n}$ be regular sequences, such that

$$
\left(f_{1}, \ldots, f_{n}\right) \subset\left(g_{1}, \ldots, g_{n}\right) .
$$

Let $f_{i}=\sum_{j=1}^{n} a_{i j} g_{j}$ for $i=1, \ldots, n$, and set $\Delta=\operatorname{det}\left(a_{i j}\right)$. Then the almost complete intersection $\left(f_{1}, \ldots, f_{n}, \Delta\right)$ is a Koszul sequence. 
Proof. The initial sequence $f_{1}, \ldots, f_{n}$ is a Koszul sequence by Example 3.5. Next we observe observe that $\left(g_{1}, \ldots, g_{n}\right)=\left(f_{1}, \ldots, f_{n}\right): \Delta$. We let $A^{(n)}$ be the Koszul complex of the sequence $f_{1}, \ldots, f_{n}$, and $M^{(n)}$ the Koszul complex of the sequence $g_{1}, \ldots, g_{n}$. Let $e_{1}, \ldots, e_{n}$ be the $R$-module basis of $A_{1}^{(n)}$ with $\partial\left(e_{i}\right)=f_{i}$ for $i=$ $1, \ldots, n$, and $h_{1}, \ldots, h_{n}$ the $R$-module basis of $M_{1}^{(n)}$ with $\partial\left(h_{i}\right)=g_{i}$ for $i=1, \ldots, n$. Then the unique algebra homomorphism $\varphi: A^{(n)} \rightarrow M^{(n)}$ with $\varphi\left(e_{i}\right)=\sum_{j=1}^{n} a_{i j} h_{j}$ for $i=1, \ldots, n$ extends the epimorphism $R /\left(f_{1}, \ldots, f_{n}\right) \rightarrow R /\left(g_{1}, \ldots, g_{n}\right)$, and

$$
\varphi\left(e_{1} \wedge \cdots \wedge e_{n}\right)=\Delta\left(h_{1} \wedge \cdots \wedge h_{n}\right) .
$$

Thus Corollary 3.3 implies that the mapping cone $C(\psi)=A^{(n)} * M^{(n)}$ with $\psi=\tilde{\varphi}$ is of Koszul type.

Note that the almost complete intersection considered in Example 3.7 is directly linked to complete intersection. More generally, let $I \subset R$ be a perfect ideal of grade $g$ in a Gorenstein ring $R, L \subset I$ a complete intersection ideal of the same grade, and $J=L: I$ the linked ideal. Then the canonical module $\omega_{A}$ of $A$ is isomorphic to $\operatorname{Hom}_{R}(R / I, R / L)$ (see for example [7]), and $\operatorname{Hom}_{R}(R / I, R / L) \cong(L: I) / L=J / L$. Therefore one obtains an exact sequence

$$
0 \longrightarrow \omega_{A} \longrightarrow R / L \longrightarrow R / J \longrightarrow 0 .
$$

The $R$-dual $A^{*}=\operatorname{Hom}_{R}(A, R)$ (with $A_{i}^{*}=\operatorname{Hom}_{R}\left(A_{g-i}, R\right)$ ) is a graded minimal free $R$-resolution of $\omega_{A}$ (cf. [7]), and since $K$ is self dual, we may lift the $R$-module homomorphism $\omega_{A} \rightarrow R / L$ to a graded complex homomorphism $\psi: A^{*} \rightarrow K^{*}$. Then the mapping cone $C(\psi)$ is a graded free resolution of $R / J$, as is well-known.

In case $R / I$ is Gorenstein, in which case $A \cong A^{*}$, one could hope to define an algebra structure on $C(\psi)$ just as in Example 3.7. But this is not possible since we would need that the composition $A \rightarrow K \rightarrow A$ is the multiplication map by an element of $R$. By rank reasons this could only be possible if $\operatorname{rank} A_{i}=\operatorname{rank} K_{i}=\left(\begin{array}{l}d \\ i\end{array}\right)$ for all $i$.

On the other hand, if we suppose that $A$ is a two-sided DG $K$-module and that the epimorphism $R / L \rightarrow R / I$ can be extended to DG $K$-module homomorphism $\varphi: K \rightarrow A$, then $\psi: A^{*} \rightarrow K^{*}$ can be chosen such that $C(\psi)$ has a natural two-sided DG $K$-module structure.

In fact, we first define the structure of a two-sided DG $K$-module on $A^{*}$ as follows: For $\alpha \in A_{i}^{*}$ and $c \in K_{j}$ we let $c \alpha, \alpha c \in A_{j}^{*}$ with $c \alpha(a)=\alpha(a \varphi(c))$ and $\alpha c(a)=\alpha(\varphi(c) a)$ for all $a \in A_{n-i-j}$. Then let $\psi=\varphi^{*}$, the $R$-dual of $\varphi$. It is then easily checked that $\psi: A^{*} \rightarrow K^{*}$ is a DG $K$-module homomorphism. This immediately implies that $C(\psi)$ is a two-sided DG $K$-module. 


\section{References}

[1] L. Avramov. Infinite free resolutions, Proc. CRM -96, Six Lectures on Commutative Algebra, Barcelona, Spain, 166, Birkhäuser, 119 - 186, 1998.

[2] A. Aramova, J. Herzog and T. Hibi. Squarefree lexsegment ideals, Math. Z. 228, 353-378 1998.

[3] E. Batzies and V. Welker. Discrete Morse theory for cellular resolutions, preprint, 2000.

[4] A. Björner, The homology and shellability of monoids and geometric lattices, In Matroid applications, 226-283, Encyclopedia of Mathematics and its applications, 40, Cambridge University Press, 1992.

[5] A. Björner and M. Wachs. Shellable nonpure complexes and posets, I, Trans. Amer. Math. Soc. 348, 1299-1327, 1996.

[6] S. Blum. Personal communication.

[7] W. Bruns and J. Herzog. Cohen-Macaulay rings, Revised Edition, Cambridge, 1996.

[8] S. Eliahou and M. Kervaire. Minimal resolutions of some monomial ideals, J. Algebra 129, 1-25, 1990.

[9] R. Fröberg. Some complex constructions with applications to Poincaré series, Séminaire d'Algèbre Paul Dubreil, Proceedings, Paris 1977-78, LNM 740, Springer-Verlag, 272- 284, 1978.

[10] G. Evans and H. Charalambous. Resolutions obtained by iterated mapping cones, J. Algebra 176, 750-754, 1995.

[11] D. Gemeda. Multiplicative structure of finite free resolutions of ideals generated by monomials in an $R$-sequence, Ph.D. Thesis, Brandeis University, 1976.

[12] G. L. Levin and L. L. Avramov. Factoring Out the Socle of a Gorenstein Ring, J. Algebra 55, 74-83, 1978.

[13] I. Novik, A. Postnikov and B. Sturmfels. Syzygies of oriented matroids, preprint 2000.

[14] V. Reiner and V. Welker. Linear syzygies of Stanley Reisner ideals, to appear in Math. Scand. 
[15] E. Sköldberg. Personal communications.

[16] D.J.A. Welsh. Matroid Theory, Academic Press, 1976.

This article may be accessed via WWW at http://www.rmi.acnet.ge/hha/ or by anonymous ftp at

ftp://ftp.rmi.acnet.ge/pub/hha/volumes/2002/n2a12/v4n2a12.(dvi,ps,pdf)

Jürgen Herzog juergen.herzog@uni-essen.de

Fachbereich Mathematik und Informatik, Universität-GHS Essen, 45117 Essen,

Germany

Yukihide Takayama takayama@se.ritsumei.ac.jp

Department of Mathematical Sciences,

Ritsumeikan University,

1-1-1 Nojihigashi, Kusatsu, Shiga 525-8577,

Japan 